\title{
Learning therapy for older adults with cognitive impairment: a pilot study
}

Mei-Yi Sze ${ }^{1}$, MSc, Stanley KF Tam ${ }^{2}$, FRCP (Lond, Edin, Glasg), Yuk-Piu Chan ${ }^{1}$, MSc, Hei-Long $\mathbf{T o}^{1}$, MSc

\begin{abstract}
Background. Studies in Japan, America and Taiwan have revealed that learning therapy, using reading aloud and arithmetic, can improve cognitive functions. The aim of the present pilot study was to investigate the effect of learning therapy on the cognitive functions and instrumental activities of daily living (IADL) performance of older adults with cognitive impairment in Hong Kong.

Methods: Outpatients of the Occupational Therapy Department of our hospital who were aged $\geq 60$ years and scored $>2$ nd and $\leq 16$ th percentile according to age- and education-adjusted cut-off in the Hong Kong version of Montreal Cognitive Assessment (HK-MoCA) were recruited between November 2017 and March 2018. Patients were assigned to the intervention or control group. Patients in the intervention group completed 30-minute reading aloud and 30-minute arithmetic sessions daily for 8 weeks. Once per week these sessions were assisted by an occupational therapist and assistant; on other days, the tasks were self-completed. Patients in the control group received the usual care including suggested structured daily routine for 8 weeks. The primary outcome measure was change in cognitive functions as measured by HK-MoCA and the Chinese version of the Mattis Dementia Rating Scale (CDRS). Secondary outcome measure was change in score of the Hong Kong Chinese version of the Lawton IADL scale. Participants were assessed before and after intervention.
\end{abstract}

Results: A total of 22 patients were assigned to the intervention group (10 women, $1 \mathrm{man}$ ) or the control group ( 8 women, 3 men). After 8 weeks, the intervention group improved significantly in terms of HKMoCA (mean change $=4.4, p=0.005$ ), specifically in the subscales of attention (mean change $=1.0, \mathrm{p}=0.04$ ), language (mean change $=2$, $\mathrm{p}=0.006$ ), abstraction (mean change $=0.64, \mathrm{p}=0.008$ ), and delayed recall (mean change $=1.55, \mathrm{p}=0.027$ ); CDRS (mean change $=11.4, \mathrm{p}=0.004$ ), specifically in the subscales of attention (mean change $=1.6, p=0.016$ ), initiation/preservation (mean change $=6.6, p=0.005$ ), and memory (mean change $=3.5, \mathrm{p}=0.012$ ); and Lawton IADL (mean change=1.0, $\mathrm{p}=0.009$ ). In contrast, in the control group, there were no significant changes.

Conclusion: The learning therapy intervention involving reading aloud and arithmetic enhanced cognitive functions and IADL performance of older adults with cognitive impairment.

Key words: Cognition; Cognitive dysfunction; Early medical intervention; Health services for the aged; Neurocognitive disorder; Rehabilitation

\section{ORIGINAL ARTICLE}

Occupational Therapy Department,

Hong Kong Buddhist Hospital

2 Department of Medicine, Hong Kong Buddhist Hospital

Correspondence to: Mei-Yi Sze, Occupational Therapy Department, Hong Kong Buddhist Hospital, Lok Fu, Kowloon, Hong Kong. Email: smy997@ha.org.hk 


\section{INTRODUCTION}

Increased life expectancy leads to increased prevalence of chronic health issues, including cognitive impairment, which is a transitional state between normal age-related cognitive decline and major neurocognitive disorder (dementia). Approximately 45.8 million people worldwide had dementia in 2015, and this is expected to increase to 131.5 million by $2050 .{ }^{1}$ In Hong Kong, approximately 103433 people had dementia in 2009, and this is expected to rise to 332688 in 2039. ${ }^{2}$ Early identification and management of cognitive impairment can help slow functional deterioration. Therefore, it is important to explore affordable and easy-to-use cognitive intervention programmes for dementia care.

Learning therapy is a cognitive training programme that uses reading aloud and simple arithmetic exercises., ${ }^{3,4}$ Neuroimaging studies have indicated that reading aloud and arithmetic activate and stimulate the frontal cortex, especially the dorsolateral prefrontal cortex, the temporal cortex, and the parietal association cortex., ${ }^{5,6}$ Randomised controlled trials have also shown that learning therapy improves executive functions and processing speed. ${ }^{7,8}$ Learning therapy has also been shown to improve inhibition, verbal episodic memory, and focus attention in older adults. ${ }^{9}$ Studies in the United States and Taiwan have also revealed that learning therapy improves cognition in older adults with dementia. ${ }^{4,10}$ Learning therapy has been evaluated for the treatment of cognitive impairment in Japan, the United States, and Taiwan. The aim of the present pilot study was to investigate the efficacy of learning therapy for older adults with cognitive impairment in Hong Kong, in terms of cognitive functions and instrumental activities of daily living (IADL) performance.

\section{METHODS}

The protocol of this study was approved by the Kowloon Central / Kowloon East Cluster Research Ethics Committee (reference: KC/KE-17-0197/ER4). The Declaration of Helsinki and International Conference on Harmonization Good Clinical Practice guidelines were observed. Written informed consent was obtained from each participant before commencing the study. Participants were allowed to withdraw at any time without any reason.

Outpatients of the Occupational Therapy Department, Hong Kong Buddhist Hospital were recruited from November 2017 to March 2018. The inclusion criteria were age $\geq 60$ years and Hong Kong version of the Montreal Cognitive Assessment (HK-MoCA) score of $>2$ nd and $\leq 16$ th percentile, according to age- and education-adjusted cut-offs. The exclusion criteria were participation in another cognitive rehabilitation programme and blindness or difficulty in reading.

Patients were assigned to either the intervention group or the control group for the 8-week study period from January to April 2018. Patients in the intervention group received an individualised 1-hour therapy session once per week, provided by an occupational therapist and an occupational therapy assistant. The therapy sessions involved a 30-minute reading aloud task and a 30-minute arithmetic task. In addition, patients were required to complete 30 minutes of reading aloud and arithmetic tasks each day for the remaining 6 days per week. The tasks were graded to fit the individual patient's current reading and arithmetic ability. In arithmetic, grading was achieved by varying the number of digits, presence of carry, format of arithmetic, and viewing time allowed. For example, in addition, the lowest level of difficulty was addition of single-digit numbers $(\mathrm{eg}, 3+1)$, whereas the highest level of difficulty was addition of three-digit numbers with carry, in a horizontal format, and with a short viewing time of $5 \mathrm{~s}$ (eg, 129+931). In reading aloud, grading was achieved by varying the length and complexity of words and sentences. The lowest level of difficulty was reading single simple words, whereas the highest level of difficulty was reading fairy tales. The reading materials included flashcards, short stories, ballads, and proverbs. Materials were selected according to the background, interest, and personal preferences of patients. To facilitate reading, different font sizes of reading materials were prepared.

Patients in the control group received their usual care and recommended structured daily routine with no additional intervention. They were informed that they were scheduled to receive learning therapy after a waiting period of 2 months.

The primary outcome measure was change in 
cognitive functions as measured by the HK-MoCA and the Chinese version of the Mattis Dementia Rating Scale (CDRS). The secondary outcome measure was change in IADL performance as assessed by the Hong Kong Chinese version of the Lawton IADL scale (Lawton IADL). Patients were assessed before and after the 8-week intervention period.

The HK-MoCA assesses cognitive functions including visuospatial/executive function, naming, memory, attention, language, abstraction, delayed recall, and orientation, with age- and educationcorrected cut-offs. A score of $>16$ th percentile indicates age-related cognitive decline only; a score of $\leq 16$ th to $>7$ th percentile indicates minor neurocognitive disorder; a score of $\leq 7$ th to $>2$ nd percentile indicates mild cognitive impairment; a score of $\leq 2 \mathrm{nd}$ percentile indicates major neurocognitive disorder or dementia. The HKMoCA is a highly reliable and valid instrument for detecting and tracking the progression of cognitive impairment associated with neurodegenerative diseases.

The CDRS is a valid and comprehensive instrument that assesses cognitive functions in four categories: attention, initiation/preservation, conceptualisation, and memory. Its total score is 144, with higher scores indicating higher cognitive functioning; maximal scores for the attention, initiation/preservation, construction, conceptualisation, and memory subscales are 37, 37, 6,39 , and 25 , respectively.

The Lawton IADL assesses nine areas: telephone use, shopping, food preparation, housekeeping, household maintenance, laundry, transportation, medication administration, and finance management. The total score is 27 , with higher scores indicating higher levels of independence in IADL.

The intervention and control groups were compared using Fisher's exact test for categorical data and the Mann-Whitney $U$ test for continuous data. Within-group difference between before and after the intervention were examined using the Wilcoxon signed rank test. A p value of $<0.05$ was considered statistically significant.

\section{RESULTS}

A total of 22 patients were assigned to either the intervention group (10 women, 1 man) or the control

TABLe 1

Demographics of older patients with cognitive impairment and baseline cognitive functions and instrumental activities of daily living before and after an 8-week learning therapy intervention or usual care

\begin{tabular}{|c|c|c|c|}
\hline Characteristics & Intervention group $(\mathrm{n}=11)^{\star}$ & Control group $(n=11)^{*}$ & $\mathrm{p}$ Value \\
\hline Sex & & & 0.586 \\
\hline Female & $10(90.9)$ & $8(72.7)$ & \\
\hline Male & $1(9.1)$ & $3(27.3)$ & \\
\hline Age, y & $73.2 \pm 6.9$ & $71.9 \pm 8.2$ & 0.519 \\
\hline Education level & & & 1.000 \\
\hline Uneducated & $0(0)$ & $0(0)$ & \\
\hline Primary school & $9(81.8)$ & $9(81.8)$ & \\
\hline High school or above & $2(18.2)$ & $2(18.2)$ & \\
\hline Mode of living & & & 1.000 \\
\hline Living alone & $1(9.1)$ & $0(0)$ & \\
\hline Living with carer(s) & $10(90.9)$ & $11(100)$ & \\
\hline Anti-dementia drug use & $0(0)$ & $0(0)$ & - \\
\hline Hong Kong version of Montreal Cognitive Assessment & $16.8 \pm 4.1$ & $16.9 \pm 4.3$ & 0.898 \\
\hline Chinese version of Mattis Dementia Rating Scale & $119.5 \pm 9.5$ & $117.4 \pm 11.4$ & 0.562 \\
\hline $\begin{array}{l}\text { Hong Kong Chinese version of the Lawton Instrumental } \\
\text { Activities of Daily Living scale }\end{array}$ & $20.1 \pm 4.0$ & $20.3 \pm 4.0$ & 0.748 \\
\hline
\end{tabular}

* Data are presented as No (\%) of participants or mean \pm standard deviation 
group (8 women and 3 men) and completed the programme. There were no significant differences between the two groups in terms of age, sex, education level, mode of living, anti-dementia drug use, and baseline HK-MoCA, CDRS, and Lawton IADL scores (TABLE $\mathbf{1}$ ).

After 8 weeks, the intervention group improved significantly in terms of HK-MoCA (mean change $=4.4, \mathrm{p}=0.005)$, specifically in the subscales of attention (mean change $=1.0, \mathrm{p}=0.04$ ), language (mean change $=2, \mathrm{p}=0.006$ ), abstraction (mean change $=0.64, \mathrm{p}=0.008$ ), and delayed recall (mean change $=1.55, \mathrm{p}=0.027$ ); CDRS (mean change $=11.4$, $\mathrm{p}=0.004)$, specifically in the subscales of attention (mean change $=1.6, \mathrm{p}=0.016$ ), initiation/preservation (mean change $=6.6, \mathrm{p}=0.005$ ), and memory (mean change $=3.5, \mathrm{p}=0.012$ ); and Lawton IADL (mean change $=1.0, \mathrm{p}=0.009)$ [TABLES 2 and 3].

In contrast, in the control group, there was no significant improvement in HK-MoCA (mean change $=-1.1, \mathrm{p}=0.233$ ), CDRS (mean change $=-3.9$, $\mathrm{p}=0.079$ ), or Lawton IADL (mean change $=-0.3$, $\mathrm{p}=0.317$ ) [TABLE 2].

\section{DISCUSSION}

Reading aloud and arithmetic involve many cognitive functions. Reading aloud requires attention to modulate the voice, and long-term memory (semantic and verbal episodic memory) to read and comprehend the text. Arithmetic requires attention and long-term memory to recognise visually presented numbers, working memory to solve arithmetic operations, and executive functions to control hand movements.

Learning therapy has been shown to improve cognitive functions of patients with cognitive impairment in Japan, the United States, and Taiwan., ${ }^{3,-10}$ Our results indicated similar positive effects of learning therapy on cognitive functions and IADL performance in older adults with cognitive impairment in Hong Kong. However, compared with previous studies, ${ }^{7-9}$ our study did not show similar improvements in terms of visuospatial performance as measured by the HK-MoCA (mean change $=0.56$, $\mathrm{p}=0.107$ ) or construction as measured by the CDRS (mean change $=-0.19, \mathrm{p}=0.317$ ). This discrepancy may be because previous studies included healthy

TABLE 2

Comparison of outcome measures between intervention and control groups before and after an 8-week learning therapy intervention or usual care

\begin{tabular}{|c|c|c|}
\hline Outcome Measures & Intervention group $(n=11)^{\star}$ & Control group $(n=11)^{\star}$ \\
\hline \multicolumn{3}{|c|}{ Hong Kong version of Montreal Cognitive Assessment } \\
\hline Pretest & $16.8 \pm 4.1$ & $16.9 \pm 4.3$ \\
\hline Posttest & $21.2 \pm 5.8$ & $15.8 \pm 4.2$ \\
\hline Change & 4.4 & -1.1 \\
\hline $\mathrm{p}$ Value & 0.005 & 0.233 \\
\hline \multicolumn{3}{|c|}{ Chinese version of Mattis Dementia Rating Scale } \\
\hline Pretest & $119.5 \pm 9.5$ & $117.4 \pm 11.4$ \\
\hline Posttest & $130.9 \pm 12$ & $113.5 \pm 13.3$ \\
\hline Change & 11.4 & -3.9 \\
\hline$p$ Value & 0.004 & 0.079 \\
\hline \multicolumn{3}{|c|}{$\begin{array}{l}\text { Hong Kong Chinese version of the Lawton Instrumental Activities of } \\
\text { Daily Living scale }\end{array}$} \\
\hline Pretest & $20.1 \pm 4.0$ & $20.3 \pm 4.0$ \\
\hline Posttest & $21.1 \pm 4$ & $20 \pm 4.6$ \\
\hline Change & 1.0 & -0.3 \\
\hline $\mathrm{p}$ Value & 0.009 & 0.317 \\
\hline
\end{tabular}

* Data are presented as mean \pm standard deviation 
TABLE 3

Changes in subscales of the Hong Kong version of Montreal Cognitive Assessment (HK-MoCA) and the Chinese version of Mattis Dementia Rating Scale (CDRS) of older patients with cognitive impairment before and after an 8-week learning therapy intervention or usual care

\begin{tabular}{|c|c|c|c|c|c|c|}
\hline \multirow[t]{2}{*}{ Instrument } & \multicolumn{3}{|c|}{ Intervention group $(n=11)$} & \multicolumn{3}{|c|}{ Control group $(n=11)$} \\
\hline & Pretest $^{*}$ & Posttest $^{\star}$ & $p$ Value & Pretest $^{\star}$ & Posttest $^{\star}$ & $\mathrm{p}$ Value \\
\hline \multicolumn{7}{|l|}{ HK-MoCA } \\
\hline Executive/visuospatial & $2.18 \pm 0.87$ & $2.73 \pm 1.42$ & 0.107 & $2.09 \pm 1.51$ & $2.18 \pm 1.60$ & 0.749 \\
\hline Naming & $2.27 \pm 0.79$ & $2.45 \pm 0.82$ & 0.157 & $1.82 \pm 0.87$ & $1.73 \pm 0.79$ & 0.317 \\
\hline Attention & $3.64 \pm 1.12$ & $4.64 \pm 1.29$ & 0.040 & $4.09 \pm 1.45$ & $3.55 \pm 1.04$ & 0.059 \\
\hline Language & $2.63 \pm 0.50$ & $4.63 \pm 1.29$ & 0.006 & $2.36 \pm 0.50$ & $2.45 \pm 0.52$ & 0.655 \\
\hline Abstraction & $0.91 \pm 0.83$ & $1.55 \pm 0.52$ & 0.008 & $0.55 \pm 0.52$ & $0.89 \pm 0.40$ & 0.180 \\
\hline Delayed recall & $0.63 \pm 1.03$ & $2.18 \pm 2.27$ & 0.027 & $1.18 \pm 1.08$ & $0.45 \pm 0.82$ & 0.038 \\
\hline Orientation & $4.55 \pm 1.57$ & $4.82 \pm 1.53$ & 0.408 & $4.82 \pm 1.08$ & $4.64 \pm 1.50$ & 0.589 \\
\hline \multicolumn{7}{|l|}{ CDRS } \\
\hline Attention & $33.7 \pm 1.19$ & $35.3 \pm 0.90$ & 0.016 & $34.5 \pm 1.44$ & $33.9 \pm 2.93$ & 0.458 \\
\hline Initiation/preservation & $26.8 \pm 5.42$ & $33.4 \pm 5.03$ & 0.005 & $26.5 \pm 5.09$ & $25.4 \pm 5.52$ & 0.105 \\
\hline Construction & $5.91 \pm 0.30$ & $5.73 \pm 0.90$ & 0.317 & $5.82 \pm 0.40$ & $5.73 \pm 0.90$ & 0.655 \\
\hline Conceptualisation & $35.5 \pm 2.46$ & $37.5 \pm 2.16$ & 0.073 & $34.5 \pm 2.54$ & $33.2 \pm 2.96$ & 0.055 \\
\hline Memory & $15.5 \pm 4.66$ & $19.0 \pm 5.57$ & 0.012 & $16.1 \pm 4.30$ & $14.9 \pm 3.36$ & 0.730 \\
\hline
\end{tabular}

* Data are presented as mean \pm standard deviation

adult or older adults, whereas the present study investigated older adults with cognitive impairment.

Although there were improvements in naming (mean change $=0.18, \mathrm{p}=0.157$ ) and orientation (mean change $=0.27, \mathrm{p}=0.408$ ) as measured by the HK-MoCA, and in conceptualisation (mean change $=2.0, \mathrm{p}=0.073$ ) as measured by the CDRS, these improvements were not significant after 8 weeks of intervention. Further studies are recommended to determine whether the improvements become significant if the intervention duration is extended.

In the present study, IADL performance improved significantly after learning therapy. This can be explained by the necessity of various cognitive functions to perform IADL. Taking cooking as an example, executive function is needed in choosing a menu, memory is needed in remembering the contents of refrigerator, and attention is needed in seasoning and cooking. Therefore, improvement in IADL performance can be extended by improvement in cognitive functions, because of the requirement of cognitive functions in IADL performance.

There are limitations to the present study. First, this was a short open-label study with a small sample size. A longer double-blinded randomised controlled study with a larger sample size is recommended to verify the results. Second, longterm effects of the intervention were not examined; a follow-up assessment at 6 months after the end of the intervention is recommended to determine the long-term maintenance effect. Third, an active control group in another type of training programme was not used. Future studies could include an active control group to verify the relative effectiveness of the intervention.

Despite the limitations, the present study extends the previous findings by showing improvements in cognitive functions and IADL performance in older adults with cognitive impairment in Hong Kong. Results of the present study have implications for clinicians when considering case management, providing more modalities for meeting the needs of older patients with cognitive impairment.

\section{CONCLUSION}

Learning therapy involving reading aloud and arithmetic improved the cognitive functions and IADL performance of older adults with cognitive 
impairment. Future large-scale studies that compare the treatment effects of learning therapy with an active control are recommended.

\section{DECLARATION}

The authors have no conflicts of interest to disclose. No sponsor was involved in the research.

\section{REFERENCES}

1. Livingston G, Sommerlad A, OrgetaV, et al. Dementia prevention, intervention, and care. Lancet 2017;390:2673-734. Crossref

2. Yu R, Chau PH, McGhee SM, et al. Trends in prevalence and mortality of dementia in elderly Hong Kong population: projections, disease burden, and implications for long-term care. Int J Alzheimers Dis 2012;2012:406852. Crossref

3. Kawashima R, Okita K, Yamazaki R, et al. Reading aloud and arithmetic calculation improve frontal function of people with dementia. J Gerontol A Biol Sci Med Sci 2005;60:380-4. Crossref

4. Kawashima R, Hiller DL, Sereda SL, et al. SAIDO learning as a cognitive intervention for dementia care: a preliminary study. J
Am Med Dir Assoc 2015;16:56-62. Crossref

5. Miura N, Watanabe J, Iwata K, et al. Cortical activation during reading of ancient versus modern Japanese texts: fMRI study. Neuroimage 2005;26:426-31. Crossref

6. Takeuchi H, TakiY, SassaY, et al. Working memory training using mental calculation impacts regional gray matter of the frontal and parietal regions. PLoS One 2011;6:e23175. Crossref

7. Uchida S, Kawashima R. Reading and solving arithmetic problems improves cognitive functions of normal aged people: a randomized controlled study. Age (Dordr) 2008;30:21-9. Crossref

8. Nouchi R, Taki Y, Takeuchi H, et al. Beneficial effects of reading aloud and solving simple arithmetic calculations (learning therapy) on a wide range of cognitive functions in the healthy elderly: study protocol for a randomized controlled trial. Trials 2012;13:32. Crossref

9. Nouchi R, TakiY, Takeuchi H, Nozawa T, Sekiguchi A, Kawashima R. Reading aloud and solving simple arithmetic calculation intervention (learning therapy) improves inhibition, verbal episodic memory, focus attention and processing speed in healthy elderly people: evidence from a randomized controlled trial. Front Hum Neurosci 2016;10:217. Crossref

10. Chen HM, Tsai LJ, Chao SY, Clark MJ. Study on the effects of individualized learning therapy on cognitive function and behavioral and psychological symptoms of dementia in the institutionalized older adults. J Nurs Res 2016;24:300-10. 